Research Article

\title{
Quantitative Characterization of Heterogeneity in Different Reservoir Spaces of Low-Permeability Sandstone Reservoirs and Its Influence on Physical Properties
}

\author{
Fengjuan Dong $\mathbb{D}^{1,2}$ Na Liu, ${ }^{3,4}$ Zhen Sun, ${ }^{5}$ Xiaolong Wei, ${ }^{6}$ Haonan Wang, \\ Junxiang Nan, ${ }^{3,4}$ and Dazhong Ren ${ }^{1,2}$ \\ ${ }^{1}$ Shaanxi Key Laboratory of Advanced Stimulation Technology for Oil \& Gas Reservoirs, Xi'an Shiyou University, Xi'an, \\ Shaanxi 710065, China \\ ${ }^{2}$ College of Petroleum Engineering, Xi'an Shiyou University, Xi'an, ShaXanxi 710065, China \\ ${ }^{3}$ Research Institute of Exploration and Development, PetroChina Changqing Oilfield Company, Xi'an, Shaanxi 710018, China \\ ${ }^{4}$ National Engineering Laboratory for Exploration and Development of Low Permeability Oil and Gas Fields, Xi'an, \\ Shaanxi, 710018, China \\ ${ }^{5}$ The Sixth Natural Gas Plant, PetroChina Changqing Oilfield Company, Yanan, Shaanxi 716000, China \\ ${ }^{6}$ Changqing Downhole Technoloogy Company, PetroChina Chuanqing Driling Engineering Company Limited, Xi'an, \\ Shaanxi 710018, China \\ ${ }^{7}$ Machine Manufacture Plant Changing Oilfield Company CNPC, Xi'an, Shaanxi 710200, China
}

Correspondence should be addressed to Fengjuan Dong; dfj_1222@126.com

Received 17 June 2021; Accepted 10 August 2021; Published 21 August 2021

Academic Editor: Xiaohu Zhang

Copyright (c) 2021 Fengjuan Dong et al. This is an open access article distributed under the Creative Commons Attribution License, which permits unrestricted use, distribution, and reproduction in any medium, provided the original work is properly cited.

\begin{abstract}
The complex pore structure of low-permeability sandstone reservoir makes it difficult to characterize the heterogeneity of pore throat. Taking the reservoir of Sanjianfang formation in QL oilfield as an example, the fractal dimension of different storage spaces is calculated by using fractal theory based on casting thin section, scanning electron microscope, and high-pressure mercury injection, and the correlation between porosity, permeability, and contribution of different storage space permeabilities is analyzed. The results show that the reservoir of Sanjianfang formation in QL oilfield mainly develops small pores, fine pores, and micropores, and the fractal dimension of micropore structure is between 2.6044 and 2.9982, with an average value of 2.8316 . The more complex the pore structure is, the stronger the microheterogeneity is. The higher the fractal dimension, the more complex the pore structure and the smaller the porosity and permeability. The fractal dimensions of small pores, fine pores, and micropores increase successively with the decrease in pore radius, and the microstructure heterogeneity of large pores is weaker than that of small pores. It provides a theoretical basis for the exploration and development of low-permeability sandstone reservoirs.
\end{abstract}

\section{Introduction}

The pore structure directly controls the reservoir and percolation characteristics and affects the oil/gas production capacity [1]. The pore structure of low-permeability sandstone reservoirs is complex, and the porosity and permeability parameters alone cannot meet the requirements for accurate description and characterization of low-permeability sandstone reservoirs. Therefore, only by studying the pore structure, can we grasp the accumulation and production rules of reservoir oil and gas from the genetic mechanism and provide technical support for the prediction of high-quality and low-permeability reservoirs and the improvement of oil and gas productivity $[2,3]$. Compared with conventional reservoirs, low-permeability sandstone reservoirs are characterized by small pore throat and low dispersion. As a result, pore throat sorting coefficient, mean coefficient, and other parameters cannot accurately reflect 
the heterogeneity of micropore structure in low-permeability sandstone reservoirs. In recent years, it is considered that reservoir pores have self-similarity in a certain scale range and belong to fractal structure $[4,5]$. The fractal dimension of capillary pressure was used to characterize the complexity of pore structure $[6,7]$. A large number of studies have shown that there is obvious heterogeneity of the pore throat structure at the micro/nanometer scale [8-11]. However, there is a lack of quantitative studies on the heterogeneity of pore distribution in porous media, and the mechanism of porous media affected by microscale pore heterogeneity needs to be further understood [12]. Taking the reservoir of Sanjianfang formation in QL oilfield as an example, the fractal dimension of different storage spaces is calculated by using fractal theory based on casting thin section, scanning electron microscope, and high-pressure mercury injection, and the correlation between porosity, permeability, and contribution of different storage space permeabilities is analyzed. It provides theoretical basis for the exploration and development of low permeability sandstone reservoirs.

\section{Methods}

2.1. Test Method. A $2.5 \mathrm{~cm}$ diameter plunger sample is drilled from the core for use in wafer grinding, physical properties, and high-pressure mercury pressure tests.

(1) Before testing, wash the sample with oil:

The sample was washed with methanol and dichloromethane mixture in the Soxhlet extractor. When the fluorescence of the washing fluid was very low and unchanged, the washing oil was considered to be finished, and the sample was dried continuously by microwave at $100^{\circ} \mathrm{C}$ for $24 \mathrm{~h}$.

(2) Casting sheet observation:

After the treatment, the samples were injected into the red casting body, and the thin slices with a thickness of $0.03 \mathrm{~mm}$ were ground. Under the polarized light microscope, the statistics and study of petrology and pores were carried out by the pointmeter method (300 points were counted for each sample). The experimental methods were strictly carried out in accordance with SY/T 5913-2004 "Rock thin section preparation" [13].

(iv) Petro physical tests:

The experimental methods were strictly carried out in accordance with SY/T6385-1999 "Test method for porosity and permeability of overburden rocks" [14]. FYKS-1 porosity-permeability tester with high temperature and overburden pressure has tested the porosity and permeability. The main technical parameters are as follows: (1) the effective pressure of simulated formation is less than $70 \mathrm{MPa}$; (2) the effective temperature of simulated formation is less than $150^{\circ} \mathrm{C}$; (3) applicable core is $\Phi 25 \times 25-80 \mathrm{~mm}$ or $\Phi 38 \times 40-80 \mathrm{~mm}$; and (4) measurement precision of permeability is less than $10 \%$ for low-permeability reservoir and is less than $5 \%$ for middle and high permeability reservoir; measurement precision of porosity is $0.5 \%$.

(v) High-pressure mercury injection experiment:

The AutoPore IV mercury injection test was conducted under the conditions of $22^{\circ} \mathrm{C}$ room temperature, $46 \%$ to $68 \%$ relative humidity, and $0.49 \mathrm{~N} / \mathrm{m}$ interfacial tension of mercury. The experimental method was strictly carried out in accordance with GB/T29171-2012 "Rock capillary pressure measurement" [15].

2.2. Fractal Theory. Fractal theory was founded in the mid1970s, and its research object is the disordered and selfsimilar system widely existing in nature and social activities $[16,17]$. Fractal dimension is a reflection of the ability of fractal to fill the embedded space, and most fractal dimensions are fractions. By combining fractal theory with the Laplace equation, the relationship between cumulative pore volume and capillary pressure can be obtained as follows:

$$
S_{H g}=1-\left(\frac{P_{c}}{P_{\min }}\right)^{D-3},
$$

where $S_{H g}$ is the volume of mercury entering the pores, \%, and $P_{\min }$ is the capillary pressure corresponding to the maximum pore throat radius, namely, the mercury inlet pressure, $\mathrm{MPa}$.

Take the logarithm of both sides of equation (1) to get the following:

$$
\lg \left(1-S_{H g}\right)=(D-3) \lg P_{c}-(D-3) \lg P_{\min }
$$

According to equation (2), the pressure-cumulative mercury intake curve can be drawn, and the fractal dimension $(D)$ can be obtained.

\section{Pore Types and Physical Properties of Reservoir}

To ensure the representativeness of experimental samples, the selection of rock samples is based on the principle of controlling the whole area, covering reservoirs with different physical properties and selecting more main gas-producing reservoirs. Through the casting thin section analysis and research on the samples taken from the reservoir of Sanjianfang formation in QL oilfield, it is concluded that the main developed reservoir spaces are intergranular pores (63.7\%), feldspar dissolution pores (15.4\%), cuttings dissolution pores $(12.5 \%)$, microfractures $(6.1 \%)$, intercrystalline pores $(0.23 \%)$, and so on. According to the analysis of reservoir physical properties and pore throat capillary pressure curves, the porosity distribution range of selected rock samples is from $11.6 \%$ to $16.7 \%$, the average value is $13.14 \%$, the permeability distribution range is from $0.395 \times 10^{-3} \mu \mathrm{m}^{2}$ to $87.695 \times 10^{-3} \mu \mathrm{m}^{2}$, and the average value is $27.513 \times 10^{-3} \mu \mathrm{m}^{2}$. The selected rock samples represent three types of reservoirs with different permeability levels, as shown in Figure 1. The value radius is more than $2.0 \mu \mathrm{m}$, and 


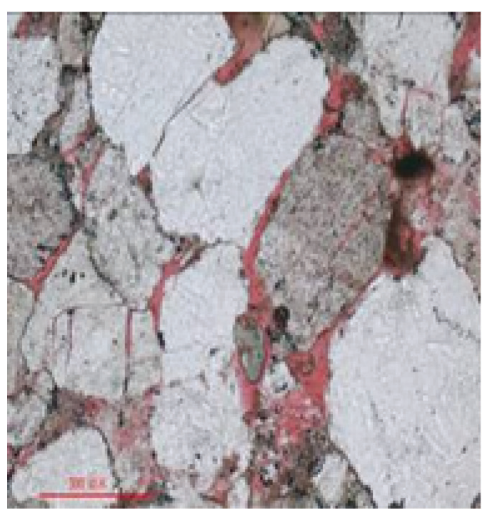

Reservoirs with permeability greater than $50 \times 10^{-3} \mu^{2}$

Sample No.10, 2567.0 m,

Development of micro fractures, intergranular pores, dissolved pores

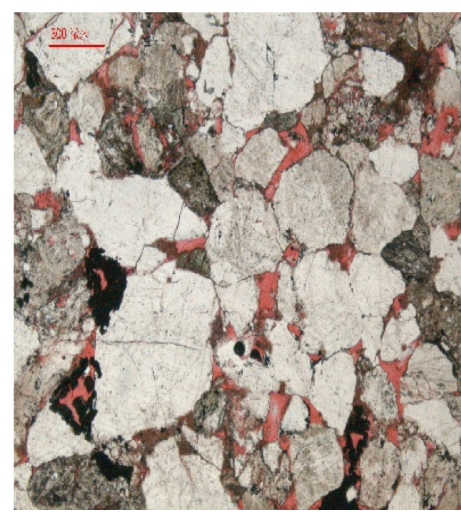

Reservoirs with permeability between $10 \times 10^{-3} \mu \mathrm{m}^{2}$ and $50 \times 10^{-3} \mu \mathrm{m}^{2}$, Sample No.5, $2480 \mathrm{~m}$, Development of dissolution pores, intergranular pores and micro pores

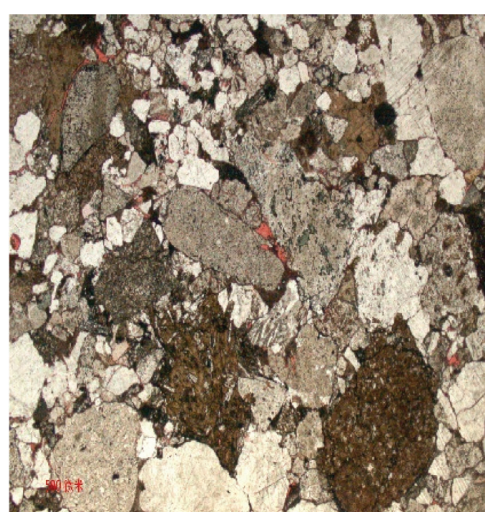

Reservoirs with permeability

between $0.1 \times 10^{-3} \mu^{2}$ and

$10 \times 10^{-3} \mu \mathrm{m}^{2}$ Sample No.3, $2712.0 \mathrm{~m}$,

Development of micro pores and dissolution pores

Figure 1: Reservoir spatial characteristics of reservoirs with different permeabilities.

the storage space is mainly composed of microcracks, intergranular pores, and dissolved pores in the reservoirs with permeability greater than $50 \times 10^{-3} \mu \mathrm{m}^{2}$. The value radius is between $0.5 \mu \mathrm{m}$ and $2.0 \mu \mathrm{m}$, and the storage space is mainly composed of dissolved pores, a few intergranular pores, and micropores in the reservoirs with permeability between $10 \times 10^{-3} \mu \mathrm{m}^{2}$ and $50 \times 10^{-3} \mu \mathrm{m}^{2}$. The value radius is between $0.05 \mu \mathrm{m}$ and $0.5 \mu \mathrm{m}$, and the storage space is mainly composed of micropores, a small number of corrosion holes in the reservoirs with permeability between $0.1 \times 10^{-3} \mu \mathrm{m}^{2}$ and $10 \times 10^{-3} \mu \mathrm{m}^{2}$.

\section{Quantitative Characterization of Heterogeneity in Different Reservoir Spaces Based on Fractal Theory}

Based on the analysis and test results of ten rock samples of Sanjianfang formation in QL oilfield, the heterogeneity of different reservoir spaces is analyzed and studied by using fractal theory.

4.1. Characteristics of Capillary Pressure Curves in Different Types of Reservoirs. The permeability of reservoir space composed of different types of pores varies greatly, which leads to significant differences in capillary pressure curves and pore distribution characteristics (Figure 2). In recent years, domestic and foreign scholars usually divide the pores into micropores ( $0 \mathrm{~nm}$ to $2 \mathrm{~nm}$ ), mesopores ( $2 \mathrm{~nm}$ to $50 \mathrm{~nm}$ ), and macropores (more than $50 \mathrm{~nm}$ ) according to their diameters [18]. However, the classification scheme is mainly applicable to shale gas reservoirs. For low-permeability sandstone reservoirs, since the molecular diameter of oil is much larger than that of gas, the pore diameter of this classification scheme is too small to be applicable. Therefore, a new classification scheme is needed to low-permeability sandstone reservoirs.
Through the analysis of the experimental results of mercury injection in ten low-permeability sandstone samples (Figure 2) [19], it can be found that there are three turning points in the mercury injection curve, and the connected pores are divided into four different scales, namely, micropores, fine pores, small pores, and mesopores. In the mercury injection curve, the three turning points occur around the pore radius of $1.0 \mu \mathrm{m}, 10.0 \mu \mathrm{m}$, and $50.0 \mu \mathrm{m}$, respectively, and the corresponding pressures are $1.0 \mathrm{MPa}, 0.08 \mathrm{MPa}$, and $0.02 \mathrm{MPa}$, respectively. Figure 2 shows the level of the three kinds of different permeability sandstone samples in mercury porosity distribution with different scales and frequency curve features. Reservoirs with permeability greater than $50 \times 10^{-3} \mu \mathrm{m}^{2}$ mainly develop intergranular pores but also develop a few microfractures, with mesopores, thick throat, and good connectivity. The discharge pressure is low, and the mercury injection curve is obvious "horizontal platform" in the process of mercury injection. The proportion of mesopore, small pore, fine pore, and micropore in this kind of reservoir is $8.02 \%, 9.73 \%, 56.88 \%$, and $25.37 \%$, respectively. Reservoirs with permeability between $10 \times 10^{-3} \mu \mathrm{m}^{2}$ and $50 \times 10^{-3} \mu \mathrm{m}^{2}$ mainly develop intergranular pores and dissolution pores, which strengthen the connectivity between pores. The displacement pressure is medium, and the mercury injection curve is weak "horizontal platform" in the process of mercury injection. This kind of reservoir is mainly composed of fine pores and micropores with a few small pores, accounting for $63.06 \%, 35.64 \%$, and $1.3 \%$, respectively. Reservoirs with permeability between $0.1 \times 10^{-3} \mu \mathrm{m}^{2}$ and $10 \times 10^{-3} \mu \mathrm{m}^{2}$ mainly develop micropores and a few dissolved pores, with narrow pores and often open "dead end pores" at one end [20], which contribute little to the permeability, and the discharge pressure is high, and the curve of mercury injection is steep in the process of mercury injection. This kind of reservoir is mainly composed of fine pores and micropores, accounting for $39.16 \%$ and $60.84 \%$, respectively.

On the whole, as the permeability decreases, the main peak of pore throat distribution shifts to the left, the 

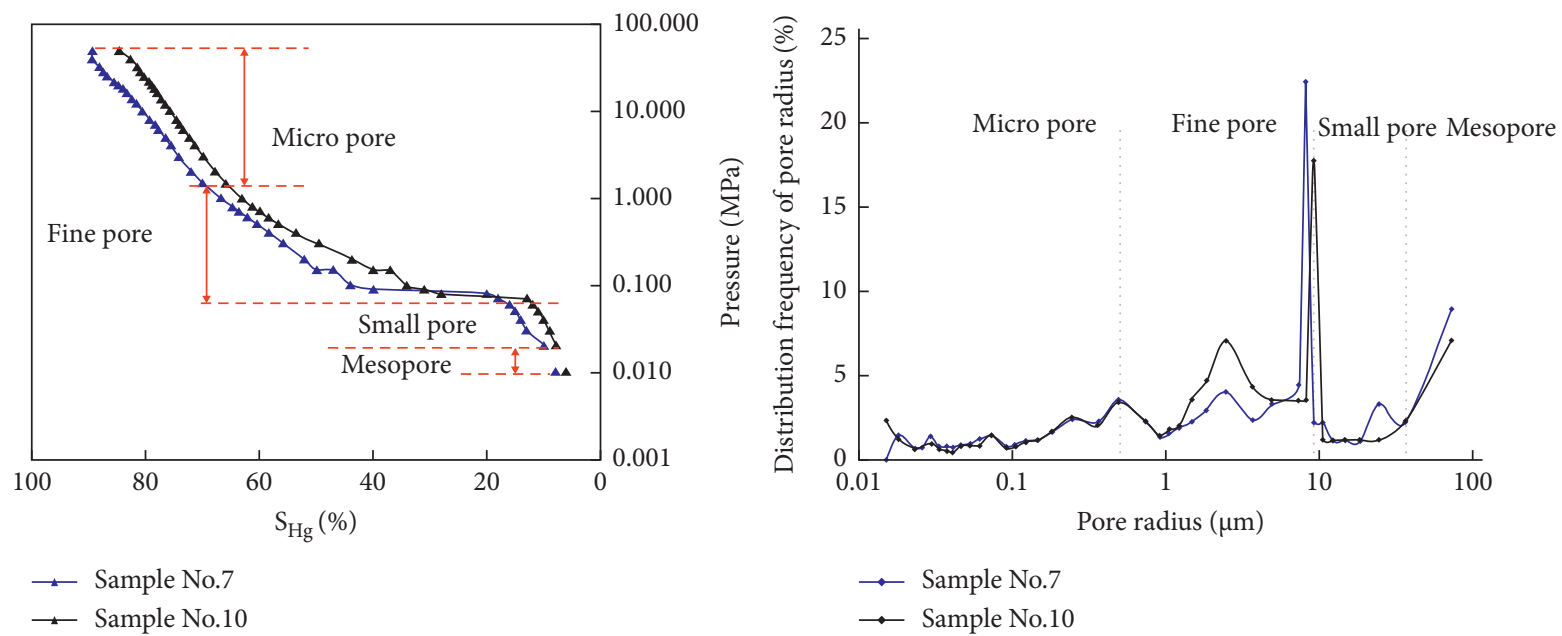

Reservoirs with permeability greater than $50 \times 10^{-3} \mu \mathrm{m}^{2}$
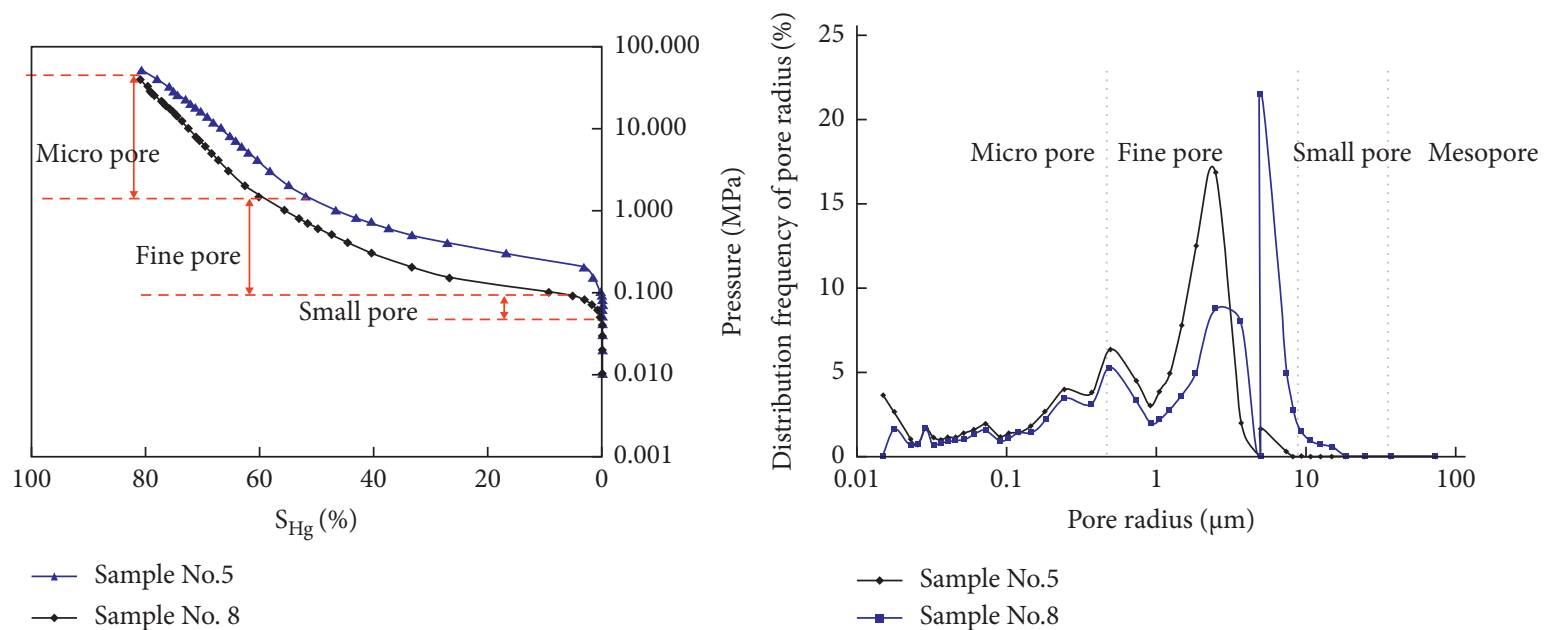

— Sample No.5

$\rightarrow$ Sample No. 8

$\rightarrow$ Sample No.8

Reservoirs with permeability between $10 \times 10^{-3} \mu \mathrm{m}^{2}$ and $50 \times 10^{-3} \mu \mathrm{m}^{2}$
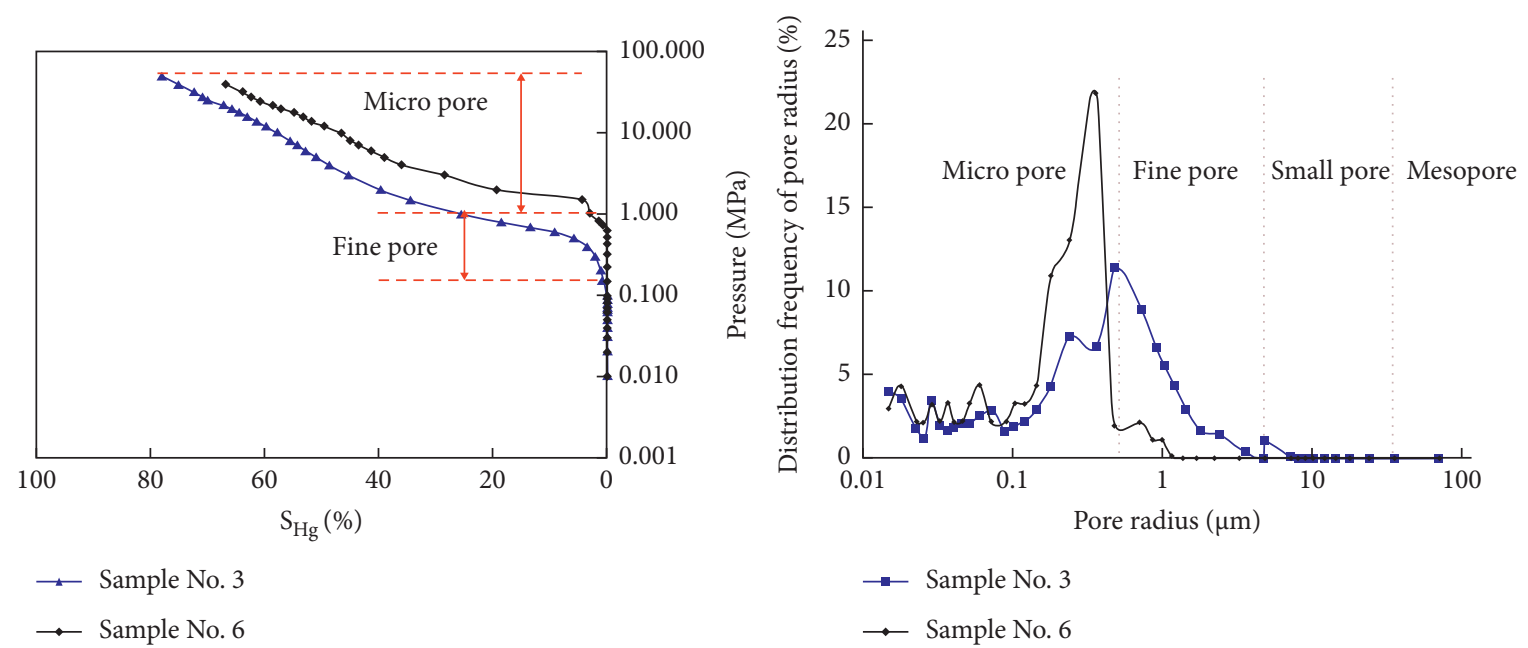

$\longrightarrow$ Sample No. 3

$\rightarrow$ Sample No. 6

Reservoirs with permeability between $0.1 \times 10^{-3} \mu \mathrm{m}^{2}$ and $10 \times 10^{-3} \mu \mathrm{m}^{2}$

FIgURE 2: Capillary pressure curves and pore distribution characteristics of different types of reservoirs. 
Table 1: Proportions and fractal dimensions of different pores.

\begin{tabular}{lcc}
\hline Pore of different scales & Proportion (\%) & Fractal dimension \\
\hline Small pore & 3.74 & 2.8109 \\
Fine pore & 45.595 & 2.8211 \\
Micropore & 49.295 & 2.8504 \\
\hline
\end{tabular}

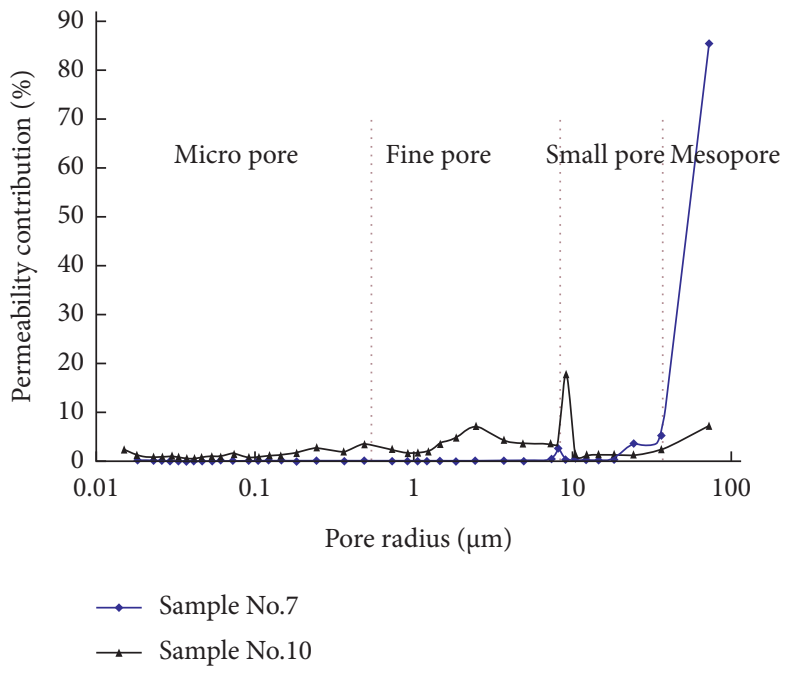

$\mathrm{K}>50 \times 10^{-3} \mu \mathrm{m}^{2}$

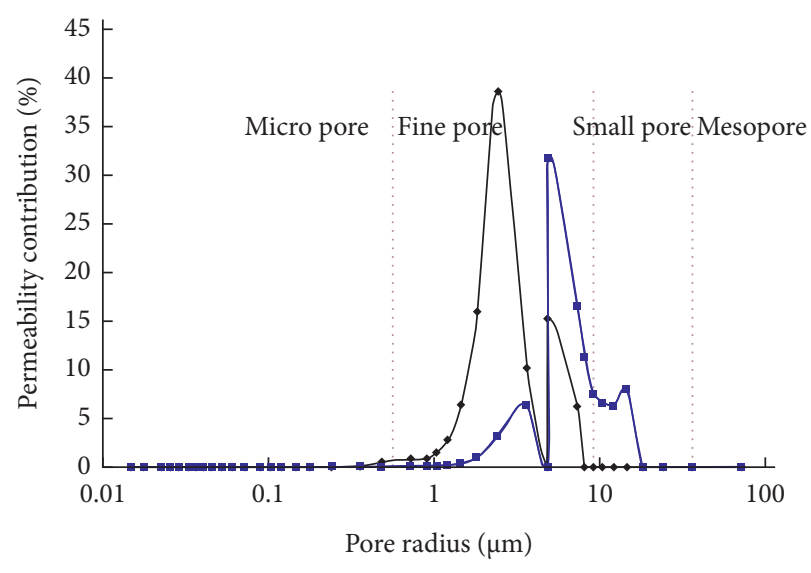

- Sample No 5

- Sample No 8

$10 \times 10^{-3} \mu \mathrm{m}^{2}<\mathrm{K}<50 \times 10^{-3} \mu \mathrm{m}^{2}$

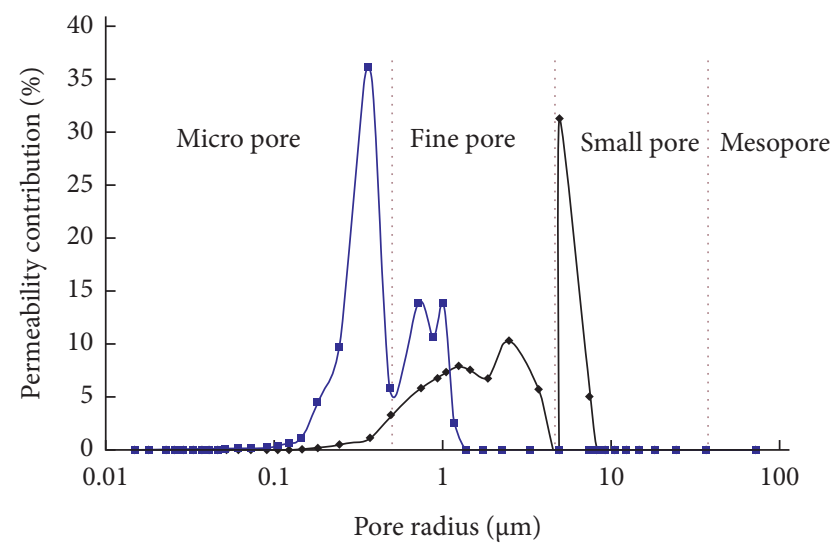

$\rightarrow$ Sample No 3
$\rightarrow-$ Sample No 6

$0.1 \times 10^{-3} \mu \mathrm{m}^{2}<\mathrm{K}<10 \times 10^{-3} \mu \mathrm{m}^{2}$

Figure 3: The permeability contribution of different pores.

distribution becomes narrower, and the morphological fluctuation of tail peak increases, which indicates that the distribution of pore throat is complex and highly heterogeneous, and various types of pore throat exist in lowpermeability sandstone reservoirs [21, 22].

4.2. Quantitative Characterization of Heterogeneity in Different Storage Spaces. Fractal theory is generally used to study irregular shapes with complex structure and selfsimilarity, which is represented by fractal dimension. For low-permeability sandstone reservoirs, the pores are only in a certain scale range, and the pore structure has fractal characteristics [23]. There are only two samples with macropores, and the distribution frequency of macropores is low, accounting for only $1.37 \%$. Therefore, macropores are not considered in this study. By statistics and analysis of the fractal dimensions of pores of different scales in ten sandstone samples, it can be seen that the fractal dimensions of small pores, fine pores, and microholes increase successively. Among them, the fractal dimension of small pores is from 2.8454 to 2.9969 , with an average of 2.8109 . The fractal dimension of fine pores is from 2.2044 to 2.9982 , with an average of 2.8211. And the structure of micropores is relatively 


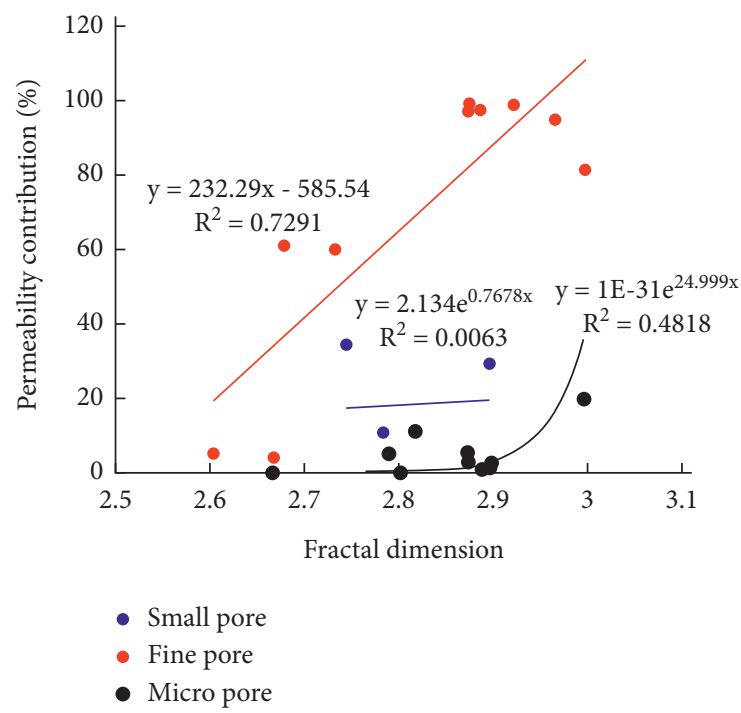

FIGURE 4: The relationship between permeability contribution and fractal dimension of different pores.
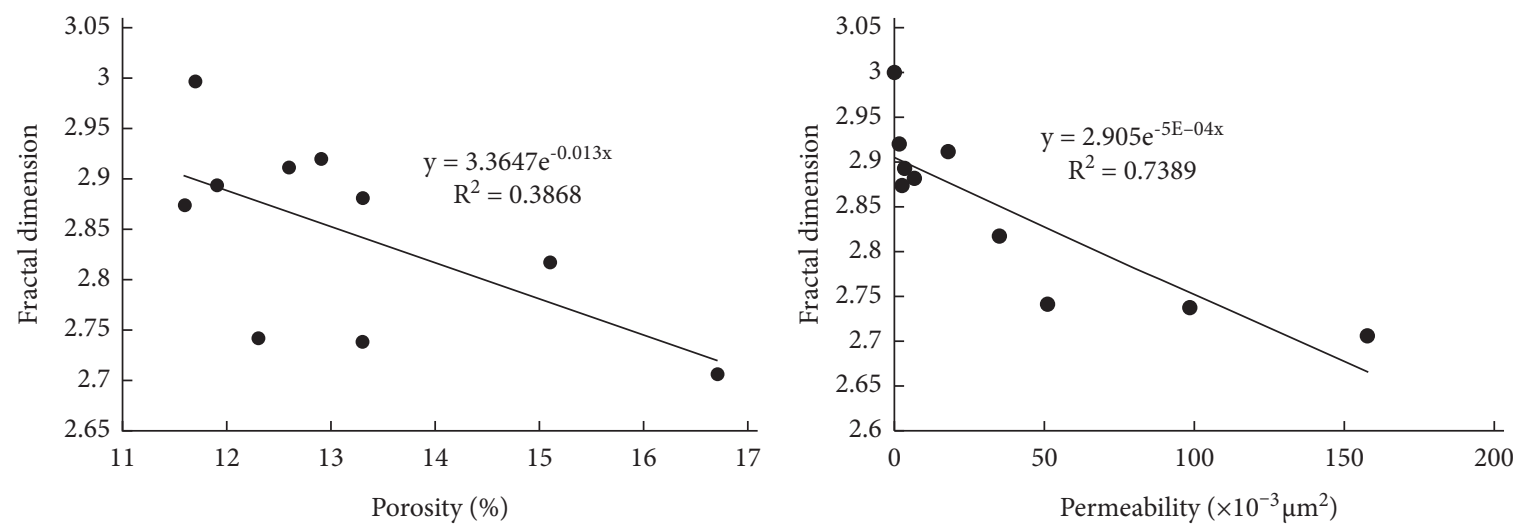

FIGURE 5: Relationship between reservoir properties and fractal dimension.

homogeneous, and the fractal dimension is from 2.666 to 2.9961 , with an average of 2.8504 . According to statistics on the relationship between the proportion of pores in different scales and the fractal dimension (Table 1), the proportion of small pores, fine pores, and micropores develop in the reservoir of the Sanjianfang formation in the study area increases successively and the fractal dimension increases which agrees with the fact that the pore structure is more complex and heterogeneous in the small pore development zone than in the large pore development zone $[24,25]$.

\section{Effects of Different Reservoir Space Heterogeneities on Reservoir Physical Properties}

5.1. Permeability Contribution of Different Reservoir Spaces. The size and distribution of pore throat are the key factors to control the properties of low-permeability sandstone reservoirs. For low-permeability sandstone reservoirs with different permeabilities, a small number of relative macropores develop to control the permeability, but relatively small pores have little effect on permeability (Figure 3). However, the contribution of relatively small pores to the reservoir property of low-permeability sandstone reservoirs cannot be ignored. For example, the maximum $\mathrm{Hg}$ saturation of sample no. 10 is $84.59 \%$. Under the control of mesopores and small pores, the permeability contribution rate reaches $95.1 \%$, but cumulative $\mathrm{Hg}$ saturation is only $13.1 \%$. Relatively small pores and micropores control the mercury saturation of $71.49 \%$, and the cumulative mercury saturation of micropores reaches $21.45 \%$.

5.2. Influence of Spatial Heterogeneity of Different Reservoirs on Permeability Contribution. By analyzing the relationship between the permeability contribution of pores at different scales and the fractal dimension (Figure 4), it can be found that the permeability contribution of small pores, fine pores, and micropores is positively correlated with the fractal dimension, and the correlation coefficient $\left(R^{2}\right)$ is 0.0051 , 0.7291 , and 0.4818 , respectively. That is to say, the positive correlation between permeability contribution of fine pores and micropores and fractal dimension is better than that of small pores. The main reason may be that the dissolution 
pores and intercrystalline pores develop in the fine pores and micropores scale interval, whose irregular shape makes the heterogeneity of pore structure stronger but may form dominant channels that play a leading role in fluid flow and have good seepage characteristics. A small number of solution holes develop in the small pore size interval which enhance the microscopic heterogeneity but are not the main controlling factor of dominant seepage channel.

\subsection{Effects of Reservoir Heterogeneity on Physical Properties.} By analyzing the relationship between porosity, permeability, and fractal dimension of pore structure (Figure 5), it is found that the reservoir physical property is negatively correlated with the fractal dimension. The larger the fractal dimension is, the more complex the pore structure is and the smaller the porosity and permeability are. There is no significant linear correlation between porosity and fractal dimension, and the correlation coefficient $\left(R^{2}\right)$ is only 0.3868 . However, the negative correlation between permeability and fractal dimension is obvious, and the correlation coefficient $\left(R^{2}\right)$ is 0.7847 .

\section{Conclusion and Understanding}

(1) Micropore structure of low-permeability sandstone reservoirs has fractal characteristics, and its heterogeneity can be characterized by fractal theory. The fractal dimension of micropore structure of Sanjianfang formation in QL oilfield is between 2.6044 and 2.9982, and the average value is 2.8316 . The more complex the pore structure is, the stronger the microheterogeneity is.

(2) Pore as the main reservoir space, the distribution characteristics of microheterogeneity at different scales can reflect the variation of reservoir space. The fractal dimensions of small pores, fine pores, and micropores increase successively with the decrease in pore radius, and the microstructure heterogeneity of large pores is weaker than that of small pores.

(3) Microscale heterogeneity is the key factor to determine the physical property and percolation mechanism of low-permeability sandstone reservoirs. The higher the fractal dimension, the more complex the pore structure and the smaller the porosity and permeability. The irregular shape of dissolution pores and intercrystalline pores develops in the fine pores and micropores scales which makes the heterogeneity of pore structure stronger but may form dominant channels, play a leading role in fluid flow, and show good seepage characteristics. A small number of solution holes develop in the small pore size interval that enhance the microscopic heterogeneity but are not the main controlling factor of dominant seepage channel. As a result, the positive correlation between permeability contribution of fine pores and micropores and the fractal dimension is better than that of small pores.

\section{Data Availability}

The data supporting the results of our study are included within the manuscript.

\section{Conflicts of Interest}

The authors declare that they have no conflicts of interest.

\section{Acknowledgments}

This work was financially supported by the National Natural Science Foundation of China (41802166), Shaanxi Provincial Key Research and Development Program (2021GY-140), Opening Foundation of Shaanxi Key Laboratory of Advanced Stimulation Technology for Oil and Gas Reservoirs (20JS120), and Open Foundation of Key Laboratory of Coal Resources Exploration and Comprehensive Utilization, Ministry of Natural Resources (KF2021-3).

\section{References}

[1] Z. T. Luo and Y. C. Wang, The Pore Structure of Reservoir, Science Press, Beijing, China, 1986.

[2] Y. N. Qiu and S. H. Xue, Appraisal Technology of Hydrocarbon Reservoirs, Petroleum Industry Press, Beijing, China, 1997.

[3] P. Luo, Y. N. Qiu, A. L. Jia, and X. S. Wang, "The present challenges of Chinese petroleum reservoir geology and research direction," Acta Sedimentologica Sinica, vol. 21, no. 1, pp. 142-147, 2003.

[4] K. W. Li and R. N. Home, "Fractal modeling of capillary pressure curves for the geysers rocks," Geothermics, vol. 35, no. 2, pp. 198-207, 2006.

[5] P. Li, M. Zheng, B. He, S. Wu, and X. Wang, "Pore throat structure and fractal characteristics of tight oil sandstone: a case study in the Ordos Basin, China," Journal of Petroleum Science and Engineering, vol. 149, pp. 1-10, 2016.

[6] S. H. Xue and S. P. Wang, "Fractal feature about the microstructure in sandstone reservoir: taking the paleogene shahejie formation in shengtuo oilfield as an example," Natural Gas Geoscience, vol. 24, pp. 886-893, 2013.

[7] L. Q. Zhang, Y. L. Ji, W. J. Ma, and H. Zhang, "Characteristics of fractional geometry and reservoir evaluation of fore mountain belt at begedashan," Journal of the University of Petroleum, China, vol. 22, no. 5, pp. 31-33, 1998.

[8] Y. Wang, W. K. Feng, R. L. Hu, and C. H. Li, "Fracture evolution and energy characteristics during marble failure under triaxial fatigue cyclic and confining pressure unloading (FC-CPU) conditions," Rock Mechanics and Rock Engineering, vol. 54, no. 2, pp. 799-818, 2021.

[9] C. Zhu, M. C. He, M. Karakus, X. H. Zhang, and Z. Guo, "The collision experiment between rolling stones of different shapes and protective cushion in open-pit mines," Journal of Mountain Science, vol. 8, no. 5, pp. 1391-1403, 2021.

[10] F. Wu, R. Gao, J. Liu, and C. Li, "New fractional variable-order creep model with short memory," Applied Mathematics and Computation, vol. 380, Article ID 125278, 2020.

[11] F. Wu, H. Zhang, Q. Zou, C. Li, J. Chen, and R. Gao, "Viscoelastic-plastic damage creep model for salt rock based on fractional derivative theory," Mechanics of Materials, vol. 150, Article ID 103600, 2020

[12] Q. X. Meng, W. Y. Xu, H. L. Wang, X. Y. Zhuang, W. C. Xie, and T. Rabczuk, "DigiSim - an open source software package 
for heterogeneous material modeling based on digital image processing," Advances in Engineering Software, vol. 148, Article ID 102836, 2020.

[13] National Development and Reform Commission, Rock Thin Section Preparation: SY/T 5913-2004, Petroleum industry press, Beijing, China, 2004.

[14] State Bureau of Petroleum and Chemical Industry, The Porosity and Permeability Measurement of Core in Net Confining Stress: SY/T6385-1999, Petroleum industry press, Beijing, China, 1999.

[15] Standardization Administration of China, Rock Capillary Pressure Measurement: GB/T29171-2012, Petroleum industry press, Beijing, China, 2012.

[16] R. F. Angulo, V. Alvarado, and H. Gonzalez, "Fractal dimensions from mercury intrusion capillary tests," in Proceedings of the SPE-23695, Society of Petroleum Engineering Conference, pp. 255-264, Caracas, Venezuela, March 1992.

[17] W. I. Friesen and R. J. Mikula, "Fractal dimensions of coal particles," Journal of Colloid and Interface Science, vol. 20, no. 1, pp. 263-271, 1987.

[18] D. H. Everett, "Manual of symbols and terminology for physicochemical quantities and units, appendixII: definitions, terminology and symbols in colloid and surface chemistry," Pure and Applied Chemistry, vol. 31, no. 4, p. 558, 1972.

[19] F. J. Dong, A Study on Reservoir Evaluation and Oil-Water Distribution at Water Cut Stage: A Case from Sanjianfang Formation of Qiuling Oilfield, Northwest University, Xi'an, China, 2010.

[20] S. Y. Xu and H. N. Li, "Evolvement of reservoir pore-throatnet and remaining oil distribution," Acta Petrolei Sinica, vol. 24, no. 4, pp. 48-53, 2003.

[21] K. L. Xi, Y. C. Cao, B. G. Haile et al., "How does the porethroat size control the reservoir quality and oiliness of tight sandstones? The case of lower Cretaceous Quantou Formation in the southern Songliao Basin, China," Marine and Petroleum Geology, vol. 76, pp. 263-271, 2016.

[22] Y. B. Yao, D. M. Liu, Y. Che, D. Z. Tang, S. H. Tang, and W. H. Huang, "Petrophysical characterization of coals by lowfield nulclear magnetic resonance (NMR)," Fuel, vol. 89, no. 7, pp. 1371-1380, 2010.

[23] K. Li, "More general capillary pressure and relative permeability models from fractal geometry," Journal of Contaminant Hydrology, vol. 111, no. 1-4, pp. 13-24, 2010.

[24] Y. H. Guo and D. F. Zhao, "Analysis of micro-scale heterogeneity characteristics in marine shale gas reservoir," Journal of China University of Mining \& Technology, vol. 44, no. 2, pp. 300-307, 2015.

[25] J. J. Li, Y. Liu, Y. J. Gao, B. Y. Cheng, F. L. Meng, and H. M. Xu, "Effects of microscopic pore structure heterogeneity on the distribution and morphology of remaining oil," Petroleum Exploration and Development, vol. 45, no. 6, pp. 1043-1052, 2018. 